\title{
A short-term n-3 DPA supplementation study in humans
}

\begin{abstract}
Purpose: Despite the detailed knowledge of the absorption and incorporation of eicosapentaenoic acid (EPA) and docosahexaenoic acid (DHA) into plasma lipids and red blood cells (RBC) in humans, very little is known about docosapentaenoic acid (DPA, 22:5 n3). The aim of this study was to investigate the uptake and incorporation of pure DPA and EPA into human plasma and RBC lipids. Methods: Ten female participants received $8 \mathrm{~g}$ of pure DPA or pure EPA in randomized crossover double-blinded manner over a 7-day period. The placebo treatment was olive oil. Blood samples were collected at days zero, four and seven, following which the plasma and RBC were separated and used for the analysis of fatty acids. Results: Supplementation with DPA significantly increased the proportions of DPA in the plasma phospholipids (PL) (by twofold) and triacylglycerol (TAG) fractions (by 2.3-fold, day 4). DPA supplementation also significantly increased the proportions of EPA in TAG (by 3.1-fold, day 4) and cholesterol ester (CE) fractions (by 2.0-fold, day 7) and of DHA in TAG fraction (by 3.1-fold, day 4). DPA proportions in RBC PL did not change following supplementation. Supplementation with EPA significantly increased the proportion of EPA in the plasma CE and PL fractions, (both by 2.7 -fold, day 4 and day 7) and in the RBC PL (by 1.9-fold, day 4 and day 7). EPA supplementation did not alter the proportions of DPA or DHA in any lipid fraction. These results showed that within day 4 of supplementation, DPA and EPA demonstrated different and specific incorporation patterns. Conclusion: The results of this short-term study suggest that DPA may act as a reservoir of the major long-chain n-3 fatty acids (LC n-3 PUFA) in humans.
\end{abstract}

Keyword: Docosahexaenoic acid (DHA); Docosapentaenoic acid (DPA); Eicosapentaenoic acid (EPA); Fatty acid metabolism; n-3 Polyunsaturated fatty acids (PUFA) 